\title{
Sommario
}

1

1.1

1.2

1.3

1.4

1.5

1.6

1.7

1.8

2

2.1

2.2

2.3

2.3.1

2.3.2

2.3.3

2.3.4

2.3.5

2.4

2.4.1

2.4.2

2.4 .3

2.4 .4

2.4 .5

2.4 .6

2.5

3

3.1

L'escatologia cosmica nella filosofia antica: Un'introduzione - 1

Dal mito ai filosofi presocratici - 1

Leucippo e Democrito - 3

Da Platone ai Peripatetici: catastrofi cicliche e immortalità cosmica - 5

La Stoà antica - 11

Epicuro - 13

Il dibattito filosofico sulla fine del mondo tra il III secolo e l'età di Lucrezio - 17

Oltre la filosofia: angosce apocalittiche tra II e I secolo a.C. - 21

II De rerum natura come testimone imprescindibile dell'escatologia epicurea -25

\section{La sezione conclusiva del primo libro -29}

Escatologia proemiale (1.62-79) -29

L'identità degli avversari di Lucrezio - 33

La sezione conclusiva del primo libro: le principali interpretazioni - 35

Introduzione - 38

Bignone, Furley e Sedley: Lucrezio come fedele traduttore di Epicuro - 40

Schmidt e Lévy: un finale anti-stoico -42

Salemme: Lucrezio contro Platone e contro Aristotele - $\mathbf{4 5}$

Bakker: il ritorno alla tesi anti-stoica -47

Gli Stoici come obbiettivo polemico del finale del primo libro -49

La difesa stoica della centralità e della stabilità del mondo - 50 L'importanza degli argomenti riportati da Plutarco - $\mathbf{5 2}$ La dottrina stoica del nutrimento dei corpi astrali $-\mathbf{5 5}$ lanua leti: la dissoluzione del mondo nel vuoto - $\mathbf{5 8}$ La natura controfattuale del finimondo dei vv. 1102-1113 - 61 Considerazioni conclusive -66

Sublimi teomachie ed epifanie: i modelli poetici dei vv. 1102$1117-68$

La sezione conclusiva del secondo libro - 71 Introduzione -74 
3.2

3.3

3.4

3.5

3.6

3.7

3.8

3.9

\section{4}

4.1

4.2

4.3

\section{5}

5.1

5.2

5.2.1

5.2.2

5.2.3

5.2.4

5.2 .5

5.3

5.3.1

5.3.2

5.4

5.4.1

5.4 .2

5.4 .3

5.4.4

5.4.5

5.4.6

5.4.7

Solmsen: Lucrezio come fedele traduttore di Epicuro - 75

Schrijvers: Lucrezio come "eclettico epigono dell'età tardo ellenistica" $\mathbf{7 7}$

Schiesaro: il ritorno alla tesi di Solmsen - 79

Le fonti di Lucrezio: un riepilogo - $\mathbf{8 0}$

Lucrezio e le teorie peripatetiche esposte in Philo aet. 55$75-84$

La fonte degli argomenti trasmessi da Filone — $\mathbf{8 6}$

Alcune conclusioni -93

Una pluralità di avversari: i passi anti-provvidenzialistici nel secondo libro -95

\section{La fine del mondo nella diade centrale - 99}

Il proemio "apocalittico" del terzo libro — 99

Sublime escatologico ed epica patriottica (3.830-842) - 101

Due "trionfi del tempo": le sezioni conclusive dei libri terzo e quarto 103

La sezione escatologica del quinto libro - 107

I vv. 91-415 del quinto libro: introduzione - 107

L'annuncio dei vv. 91-109: un'apertura retorica — 108

Il rovesciamento di un motivo topico: l'osservazione del cosmo 109

La tripartizione del mundus e la "maschera" empedoclea — 110

Il motivo topico dell'una dies (v. 95) - 112

Il senso della machina mundi lucreziana (v. 96) - 113

La nozione polemica di fortuna gubernans (v. 108) - 116

I vv. 110-121: la Gigantomachia filosofica intorno all'eternità del mondo 118

I nuovi Giganti - 121

La ripresa degli Aratea ciceroniani - 122

I vv. 122-234: Lucrezio contro il creazionismo e il provvidenzialismo 125

Introduzione: gli obbiettivi polemici del poeta - 125

I vv. 122-145: il corpo vitaliter animatus del mondo - 126

I vv. 146-155: le sedi degli dèi — 131

I vv. 156-194: la creazione del mondo - 133

L'attacco lucreziano al mito del Timeo — 136

Paralleli epicurei in favore di una polemica anti-stoica — 138

I vv. 174-180: uno spunto lucreziano contro il pessimismo - 146 
5.4.8 I vv. 195-234: la culpa naturae - 148

5.4.9 Lucrezio e il dibattito a lui contemporaneo sulla provvidenzialità del mondo 156

5.4.10 La misera condizione dell'uomo: i vetusta placita del pessimismo antico 160

5.4.11 Gli obbiettivi polemici dei vv. 122-234: un bilancio - 162

5.5 La seconda parte della sezione escatologica del quinto libro: $i$ vv. $235-379-164$

5.5.1 Introduzione: una risposta epicurea alle tesi di Teofrasto in Philo aet. $117-149-164$

5.5.2 La mortalità degli elementi cosmici (vv. 235-323) - 166

5.5.3 La brevità della storia umana (vv. 324-350) - 175

5.5.4 L'età del mondo - 179

5.5.5 Oltre Epicuro: la rielaborazione di Lucrezio - 180

5.5.6 La parentesi dei vv. 351-379-183

5.6 Lucrezio e i grandi miti escatologici (vv. 380-415) 186

5.6.1 Fetonte $\mathbf{1 8 8}$

5.6.2 Le fonti poetiche: il Fetonte di Euripide - 190

5.6.3 II racconto di Diodoro Siculo — 191

5.6.4 Il diluvio lucreziano - 194

5.6.5 Alcune conclusioni 195

5.7 La struttura complessiva dei vv. 91-415-196

5.8 Lo specchio del finimondo: la cosmogonia lucreziana (vv. 416508) 199

$6 \quad$ La fine del mondo nel sesto libro -202

6.1 Introduzione: il ritorno della tematica escatologica - 202

6.2 Amplificatio e nec mirum: due opposte tensioni - 203

6.3 Fonti e obbiettivi polemici dei passi escatologici nel sesto libro -206

6.4 L'immaginario escatologico relativo ai fenomeni del tuono, del lampo e del fulmine $-\mathbf{2 0 8}$

6.5 I fenomeni sismici (vv. 535-607) - 212

$6.6 \quad$ I vulcani (vv. 639-702) - 220

6.7 Gli avversari di Lucrezio nel sesto libro $-\mathbf{2 2 7}$

6.8 Le molteplici cause della fine del mondo - 232

6.9 Fine della città e fine del mondo: simboli di escatologia cosmica nella Peste di Atene $\mathbf{2 3 8}$ 
$7 \quad$ Conclusione -244

7.1 Gli obbiettivi polemici di Lucrezio: un bilancio - 244

7.2 Il sublime escatologico nel poema lucreziano - 252

7.2.1 La dialettica tra motus auctifici e motus exitiales nei proemi e nelle conclusioni - 256

7.2.2 La dialettica tra verità ed opinione: prospettiva soggettiva e prospettiva cosmica - $\mathbf{2 5 9}$

7.2.3 II "sistema" del sublime lucreziano - 261

7.2.4 L'aspetto mirabilis del mondo e l'aspirazione all'assenza di stupore -266

$8 \quad$ Appendici -271

8.1 Appendice 1. Lucr. 2.1164-1174: analisi stilistica di una conclusione "comico-diatribica" — 271

8.1.1 Introduzione - 271

8.1.2 Caput quassans (v. 1164) -274

8.1.3 In cassum [...] cecidisse (v. 1165) - 275

8.1.4 Laudat fortunas parentis (v. 1167) -276

8.1.5 Vetulae vitis (v. 1168) - 277

8.1.6 Temporis incusat momen saeclumque fatigat (v. 1169) 278

8.1.7 Crepat (v. 1170) - 279

8.1.8 Antiquum genus pietate repletum (v. 1170) - 279

8.1.9 Perfacile angustis tolerarit finibus aevum (v. 1171) -280

8.1.10 Cum minor esset agri multo modus ante viritim (v. 1172) - 281

8.1.11 Nec tenet omnia paulatim tabescere (v. 1173) - 282

8.1.12 Spatio aetatis defessa vetusto (v. 1174) - 283

8.1.13 Ire ad capulum (v. 1174) - 284

8.1.14 Lucrezio e il dibattito tardo-repubblicano sulla vetustas della terra - 286

8.2 Appendice 2. Empedocle nei passi escatologici lucreziani - 289

8.2.1 La rinascita del modello empedocleo: l'anti-Arato $2 \mathbf{2 8 9}$

8.2.2 Libro I: Empedocle e Tifeo 291

8.2.3 Empedocle nel finale del primo e del secondo libro -294

8.2.4 Il proemio della sezione escatologica nel quinto libro - 296

8.2.5 Libro VI: oltre il mirum empedocleo - 297

8.2.6 La Peste come "trionfo della Discordia": una conclusione empedoclea? - 298

8.2.7 “Angoscia dell'influenza” lucreziana: emulare Empedocle — $\mathbf{3 0 1}$ 
8.3 Appendice 3. "Finimondo con spettatore": il significato dell'escatologia lucreziana $-\mathbf{3 0 2}$

8.3.1 Introduzione - $\mathbf{3 0 2}$

8.3.2 I contadini del secondo libro e l'induperator del quinto: Roma al centro della catastrofe - $\mathbf{3 0 5}$

8.3.3 Dall'avocatio epicurea alla “terapia” lucreziana - $\mathbf{3 0 7}$

8.4 Appendice 4. Il trattato pseudo-aristotelico De mundo. Una fonte di Lucrezio? 309

8.4.1 Introduzione - $\mathbf{3 0 9}$

8.4.2 Il volo dello spirito e l'elogio della filosofia - $\mathbf{3 1 0}$

8.4.3 Una citazione condivisa - $\mathbf{3 1 5}$

8.4.4 L'aeterna salus del mondo - 316

8.4.5 L'isonomia cosmica - $\mathbf{3 1 6}$

8.4.6 Meccanismi analogici e metafore condivise - $\mathbf{3 1 8}$

8.4.7 Alle radici del "finimondo con spettatore" - $\mathbf{3 2 0}$

8.4.8 Conclusione - $\mathbf{3 2 1}$

8.5 Appendice 5. Glossario escatologico lucreziano - 323

Index locorum -342

Index rerum -354

Index nominum antiquorum -358

Index virorum mulierumque doctorum -361

Index Graecitatis - 363

Bibliografia -370 
